\title{
Finite Element Implementation of State Variable-Based Viscoplasticity Models
}

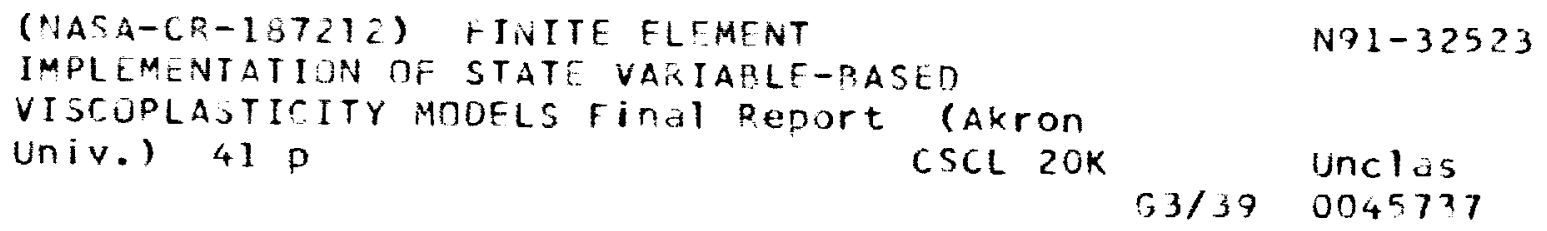

I. Iskovitz, T.Y.P. Chang, and A.F. Saleeb

University of Akron

Akron, Ohio

September 1991

Prepared for

Lewis Research Center

Under Grant NAG3-901

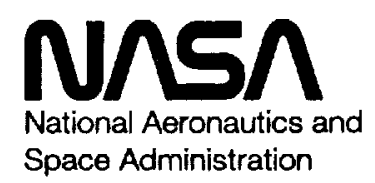


Finite Element Implementation of State Variable-Based Viscoplasticity Models

\author{
I. Iskovitz, T.Y.P. Chang and A.F. Saleeb \\ Department of Civil Engineering \\ University of Akron \\ Akron, Ohio 44325
}

\begin{abstract}
Implementation of state variable-based viscoplasticity models is made in a general purpose finite element code for structural applications of metals deformed at elevated temperature. Two constitutive models, i.e. Walker's and Robinson's models, are studied in conjunction with two implicit integration methods: the trapezoidal rule with Newton-Raphson iterations and an asymptotic integration algorithm. A comparison is made between the two integration methods, and the latter method appears to be computationally more appealing in terms of numerical accuracy and CPU time. However, in order to make the asymptotic algorithm robust, it is necessary to include a self adaptive scheme with subincremental step control and error checking of the Jacobian matrix at the integration points. Three examples are given to illustrate the numerical aspects of the integration methods tested.
\end{abstract}




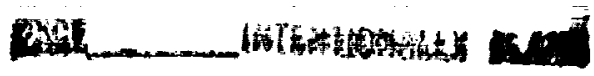




\section{Introduction}

In the design of structural components, such as jet engines or nuclear primary vessels, inelastic deformations and ratchetting effects under thermomechanical cyclings are of major concern in predicting the service lifes of such structures. To reliably predict the mechanical response of metals or composite materials at elevated temperatures, it appears that constitutive relations in the framework of a unified viscoplasticity theory are most effective. Indeed, such constitutive models can represent typical deformation phenomena, e.g. creep-plasticity interactions, cyclic strain softening or hardening, and thermal ratchetting, none of which can be properly described by the classical creep and plasticity theories. Although the viscoplastic theories are very useful in predicting the deformation behavior of high-temperature materials, they present considerable difficulties when the associated differential equations are to be solved by numerical means. This is directly related to the "stiff" nature of the nonlinear differential equations, for which a small change of a state variable may result in large changes of others.

In the literature, a great deal of study on numerical methods for integrating viscoplastic rate equations in relation to the solution of boundary value problems has been reported [1-3]. Several numerical schemes have proven to be quite effective [4]. In general terms, there are one-step methods verse multi-step methods, such as those due to Gear [5]. It has been argued that although the multi-step methods generally give better solution accuracy as well as reliability, they are not suitable for large-scale finite element analysis because of their excessive demand in computer storage. Among the one-step integration schemes, two classes of numerical methods can be identified: explicit and implicit schemes. Considerable discussion on the advantages and disadvantages of these two methods is available, e.g. $[1,2]$. No attempt will be made in the present paper to duplicate what is already available in the literature. Nevertheless, two observations may be offered: 1) the use of an explicit scheme in conjunction with self-adaptive time stepping can be very effective, however the solution accuracy may vary with the accuracy of error check embeded in the method, and 2) an implicit scheme together with iterations is generally more reliable, but requires intensive computational effort.

More recently, an asymptotic integration algorithm was proposed by Walker, et al. [6-8], where the constitutive rate equations are cast into an integral form. The integrands of solution are then expanded into Taylor series 
in a time interval, say $t \in\left[t_{n}, t_{n}+\Delta t\right]$, so that the resulting equations can be integrated term by term. When the series expansion is done about the upper limit $\left[t_{n}+\Delta t\right]$, it gives an implicit iterative scheme and the numerical solution is unconditionally stable provided that the corresponding solution is exponentially decaying [7]. One apparent advantage of this method is that the numerical scheme involves the solution of only a $3 \times 3$ matrix of equations as opposed to a $13 \times 13$ (for $2 \mathrm{D}$ problems) or a $19 \times 19$ (for $3 \mathrm{D}$ problems) matrix of equations in the case of other implicit schemes, such as the trapezoidal rule with Newton-Raphson iterations [3,9]. From a computational standpoint, the asymptotic integration algorithm appears to be quite appealing. Nevertheless, the method is relatively new and it has not been tested on various viscoplastic models. One of the objectives of this paper is to conduct a comparative study between two, one-step, implicit methods, i.e. the asymptotic integration method and the trapezoidal method with Newton-Raphson iterations. The numerical schemes will be tested on two constitutive theories: Walker's [10] and Robinson's models [11] for isotropic materials. Moreover, another objective of the present paper is to show how the viscoplastic models and the associated integration schemes can be conveniently implemented into a finite element code for structural analysis. Three numerical examples are included in this paper.

\section{Constitutive Models}

The present paper is concerned with the thermo-mechanical behavior of polycrystalline metals and similar alloys deformed at elevated temperatures. In this connection, inelastic phenomena such as creep-plasticity interactions, cyclic strain-hardening or softening, and thermal ratchetting are of primary interest in a structural analysis. Although a number of viscoplasticity theories are available to represent the aforementioned phenomena, see e.g. [12], our attention is focused on a class of state-variable based viscoplastic models, that are based on a phenomenological approach, as discussed by Freed and Chaboche [13].

In the present study, the material is assumed to be initially homogeneous and isotropic. Furthermore, the analysis is limited to small deformations. Referring to a rectangular Cartesian coordinate system $x_{i}, i=1,2,3$, the total strain rate at a material point in a deformable body is given by 


$$
\dot{\varepsilon}=\dot{\varepsilon}^{e}+\dot{\varepsilon}^{I}+\alpha \dot{T}
$$

with the incompressibility condition in inelastic strains

$$
\operatorname{tr}\left(\dot{\varepsilon}^{\mathrm{I}}\right)=0
$$

The relation between the elastic strain and the Cauchy stress rates is given by the Hooke's law:

$$
\dot{\sigma}=\mathrm{D}:\left(\dot{\varepsilon}-\dot{\varepsilon}^{I}-\alpha \dot{T}\right)
$$

where $\varepsilon$ is the total strain, $\xi^{e}$ is the elastic part of the strain, $Q$ is the matrix of thermal coefficients, $T$ is the temperature, $g$ is the Cauchy stress and D is the elastic stiffness matrix.

To represent the inelastic process of a viscoplastic material, two sets of laws are being used: 1) a flow law which governs the rate of inelastic strain as a function of the current deformation state of a body, and 2) a set of evolutional equations that defines the rate of change in the internal state variables. The framework of these laws are outlined as below. 
Flow Law:

$$
\dot{\xi}^{I}=f\left(\frac{\Sigma}{k}\right) \Sigma
$$

where $f$ represents a plastic scalar function, $\underset{\sim}{\boldsymbol{N}}$ is the effective deviatoric stress and $a$ is the back stress.

\section{Evolution Equations:}

Evolution equations describe the change of internal state during the deformation process. In general, three types of state variables can be identified: back stress, drag strength and yield strength $[9,10]$. At present, we shall consider viscoplastic models that do not have an explicit yield surface. Moreover, the change of state variables is described by two competing mechanisms: hardening vs. recovery processes (including both dynamic and static recovery). With the aforementioned considerations in mind, evolution equations may be expressed for the back stress and drag strength, respectively, in the form:

$$
\begin{gathered}
\dot{a}=H_{a} \dot{\varepsilon}_{\stackrel{\sim}{I}}^{I}-R_{a} \underset{\sim}{a} \\
\dot{k}=H_{k}\left\|\dot{\varepsilon}_{\sim}^{I}\right\|_{2}-R_{k} k
\end{gathered}
$$

where $\underset{\sim}{a}$ designates the back stress, a tensor, $k$ is the drag strength, a scalr quantity, $H$ represents a hardening function and $R$ is a recovery function.

For later discussion, both (5) and (6) may be combined into one representative form:

$$
\dot{\sim}=H_{\Omega}: \dot{\tilde{\varepsilon}}^{I}-R_{\Omega}: \underset{\sim}{\Omega}
$$

where $\Omega=(\underset{\sim}{a}, k)$.

In addition, we define the following quantities: the deviatoric components of stress are

$$
S=g-\frac{1}{3} \operatorname{tr}(\sigma)
$$

The second invariants associated with the effective and the back stresses, respectively, are: 


$$
\begin{gathered}
J_{2}=\frac{1}{2}(\underset{\sim}{\Sigma}: \underset{\sim}{\Sigma}) \\
I_{2}=\frac{1}{2}(a ; q)
\end{gathered}
$$

The material is assumed to behave thermo-elastically in hydrostatic response,

$$
\operatorname{tr}(\dot{g})=\left(\lambda+\frac{2}{3} \mu\right)[\operatorname{tr}(\dot{\xi})-3 \alpha \dot{\mathrm{T}}]
$$

where $\lambda$ is the Lame constant, $\mu$ is the shear modulus and $\alpha$ is the coefficient of thermal expansion.

For computational purposes, we have selected two specific viscoplastic models for finite element implementation. These are Walker's [9] and Robinson's models [10] for isotropic materials. The basic difference between the two models is their mathematical form. Walker's model prescibes fairly smooth behavior in the state space. On the other hand, Robinson's equations contain mathematical discontinuities in various regions of state space which require special care in numerical integration. Both models are briefly summarized below.

\subsection{Walker's Model}

Elow Jaw:

$$
\dot{\varepsilon}^{I}(t)=f \underset{\sim}{\Sigma}
$$

with

$$
f=\frac{1}{k}\left[\frac{\sqrt{J_{2}}}{k}\right]^{n-1}
$$

Evolution equations:

$$
\begin{gathered}
\dot{a}(t)=\left(n_{1}+n_{2}\right) \dot{\varepsilon}^{I}-\left[a(t)-n_{1} \varepsilon^{I}(t)\right]\left[\left(n_{3}+n_{4} e^{-n_{5} R}\right) \dot{R}+n_{6}\left(\frac{2}{3} I_{2}\right)^{\frac{m-1}{3}}\right] \\
\dot{k}(t)=n_{7} \dot{R}-n_{8} \dot{R}\left[k(t)-k_{0}\right]-n_{9}\left[k(t)-k_{0}\right]^{p}
\end{gathered}
$$


In the above, $\dot{R}$ is the magnitude of inelastic strain rate defined by:

$$
\dot{R}=\sqrt{\frac{2}{3} \dot{\xi}^{I}: \dot{\varepsilon}^{I}}
$$

and the effective stress is

$$
\underset{\sim}{\Sigma}=\frac{3}{2} S-\underset{\sim}{a}
$$

where $n, n_{1}, n_{2}, n_{3}, n_{4}, n_{5}, n_{6}, n_{7}, p, k_{0}$ are material constants, which are defined in [10].

\subsection{Robinson's Model}

In order to capture very different viscoplastic responses of a material under different loading paths, several discontinuous functions were introduced in Robinson's model, which was derived from an assumed plastic potential. This model constitutes an inelastic flow law and one evolution equation as follows.

Flow law:

$$
\underset{\sim}{\dot{\varepsilon}^{I}}=\frac{P(x) \Phi_{F}(T)}{2 r k_{T} \sqrt{J_{2}}}<F>^{n} \underset{\sim}{\Sigma}
$$

where

$$
\underset{\sim}{\Sigma}=\underset{\sim}{S}-\underset{\sim}{a}
$$

In the above, $P$ is a spline function defined in the stress or strain space [11], which provides a smooth transition from one set of material equations to another; $F$ and $G$ are material functions, also given in [11].

\section{Evolution equation:}

$$
\underset{\sim}{\dot{a}}=\frac{H_{a}}{G^{\beta}} \dot{\varepsilon}^{I}-\frac{R_{a} \Phi_{G}(T)}{k_{T} \sqrt{I_{2}}} G^{(m-\beta)} \underset{\sim}{a}
$$


where $k_{T}, n, r, m, \beta, R_{a}$ and $H_{a}$ are material constants to be determined experimentally and $\Phi(T)$ is a function of the temperature to account for temperature dependence of material properties [11].

\section{Numerical Procedures}

With the constitutive equations outlined in the preceeding section, one may proceed to the structural analysis for viscoplastic materials using the finite element method. In this context, two solution approaches are possible: the initial strain method and the tangent stiffness method. In the former approach, the viscoplastic deformations are treated as the initial strains on the right hand side of the incremental equilibrium equations. The major advantage of this method is that the global stiffness matrix remains constant under isothermal condition but the convergence rate of the corresponding iterative solution is at best linear. As an alternative, it is possible to derive the tangent material stiffness for a viscoplastic model $[12,14,15]$ so that a tangent stiffness method can be pursued. In this context, the global stiffness matrix has to be reformed for each loading increment, but the iterative solution is quadratic in terms of its convergence rate. The, choice between these two methods is largely hinged upon the coding convenience. In our analysis, we have chosen the initial strain method.

The finite element viscoplastic analysis generally involves two levels of numerical computations: global equilibrium and local constitutive calculations. At the global (or structural) level, the targeted solution time is divided into a number of time intervals and the incremental equilibrium equations are solved for each time step in succession. At the local level (or material point), the constitutive rate equations are integrated by a numerical scheme where the stress and strain rates are converted into incremental quantities. As a result, the rate form of the constitutive equations in (3) for a typical time step $t^{\prime} \in[t, t+\Delta t]$ is written as

$$
\Delta \sigma=\mathrm{D}:\left(\Delta \underline{\varepsilon}-\Delta \varepsilon^{I}-\alpha \Delta T\right)
$$

where the vector of incremental stresses are obtained from

$$
\Delta \underset{\sim}{\sigma}=\int_{t}^{t+\Delta t} \dot{\sigma} d t
$$


The expressions of $\Delta \varepsilon, \Delta \varepsilon^{I}$ and $\Delta T$ follow similar definitions.

The main objective of our analysis is to integrate the rate equations (1) (4) and (7) in an efficient way. Due to the "stiff" nature and the nonlinearity of viscoplastic rate equations, the solution accuracy of $\Delta \varepsilon_{\tilde{\varepsilon}}^{I}$ is very sensitive to the values of the state variables evaluated at the material sampling points. Therefore, an accurate and reliable numerical scheme is essential to integrate the aforementioned rate equations.

For the sake of brevity, we write (1)-(4) and (7) in the condensed form

$$
\dot{y}=\underset{\sim}{f}(\underset{\sim}{y}, t)
$$

The above equation represents a system of nonlinear, first-order, ordinary differential equations, where $y=\left(g, a, \varepsilon^{I}, k\right)$. For a three-dimensional problem, $y$ represents a vector of 19 -components, i.e. 6-Cauchy stresses, 6 back stresses, 6 inelastic strains and 1 drag strength. For a 2D problem, $y$ contains 13 components.

A number of numerical integration schemes are available to integrate (23). The choice of a particular scheme is dominated by three considerations: 1) suitability for finite element implementation, 2) solution accuracy and reliability and 3) computational cost. For instance, Gear's multi-step methods [5] are known to be very effective for integrating nonlinear differential equations such as (23) for viscoplastic materials under homogeneous deformation. However, these methods are not particular suitable for large-scale finite element computations for two reasons: 1) the methods require extensive central memory and 2) a special start-up procedure is needed. In view of this, one-step integration methods are much more desirable.

In the context of one-step integration methods, two classes of algorithms can be identified: explicit and implicit algorithms. The explicit algorithms (although less computation effort is required) are not very reliable, especially in dealing with thermo-mechanical cyclic loadings. For added solution accuracy, we have chosen the implicit algorithms. In particular, we select the trapezoidal rule with Newton-Raphson iterations and an asymptotic algorithm proposed by Walker [6,7]. Both algorithms are briefly outlined in the following subsections. 


\subsection{Implicit Trapezoidal Rule}

We consider a typical time interval $\tau \in[t, t+\Delta t]$. The trapezoidal rule can be easily obtained by using Taylor series expansion of the rates $\dot{y}$ between succesive iterations at time points $t$ and $t+\Delta t$. For discussion purposes, we introduce the following notations: $y_{n}=y(t), y_{n+1}^{i}=y(t+\Delta t)$ corresponding to the $\mathrm{i}$-th iteration. Thus, the trapezoidal rule with Newton-Raphson iterations prescribes $[3,9,18,19]$ :

$$
\left[\mathrm{I}-\frac{\Delta t}{2}{\frac{\partial f}{\partial \underset{\sim}{y}}}^{(i)}\right] \cdot \Delta{\underset{\sim}{y}}^{(i)}=\underbrace{}_{n}-{\underset{\sim}{n+1}}_{n+1}^{i}+\frac{\Delta t}{2}\left[\underline{\sim}_{n}+{\underset{\sim}{f}}_{n+1}^{(i)}\right]
$$

with the recursive relationship

$$
y_{n+1}^{(i+1)}=y_{n+1}^{i}+\Delta y^{i}
$$

where $(\partial f / \partial y)^{i}$ is a Jacobian matrix evaluated at the time $t+\Delta t$ for the $\mathrm{i}$-th iteration. The size of the Jacobian matrix is: $13 \times 13$ for $2 \mathrm{D}$ problems, and $19 \times 19$ for $3 \mathrm{D}$ problems.

It is known that stability of this algorithm is assured by its implicitness[3,9]. However, some comments are needed on the uniqueness of the solution scheme. In order to assure the uniqueness of $\Delta y$ in (24), it is necessary to impose a condition that the $p^{\text {th }}$ norm of the Jacobian matrix must always be less than one. $p$ may be any norm as long as it satisfies the basic matrix relationships $[3,5,18,19]$.

We note again that (24) represents a system of unsymmetric simultaneous equations which have to be solved at every integration point of each finite element. For a sizable finite element mesh (say 1000 elements) in threedimensions, the computation efforts required to solve these equations can be quite intensive.

\subsection{Uniformly Valid Asymptotic Algorithm}

The basic idea behind the algorithm proposed by Walker $[6,7]$ is to transform the differential equations into a set of integral equations, which can then be solved approximately using a recursive relationship. In order to evaluate the resulting integral expression, an asymptotic expansion of the related integrand is performed about the upper limit of the time interval $[t, t+\Delta t]$, resulting in an implicit integration scheme. The main advantage 
of this method is that only a $2 \times 2$ (or for certain viscoplastic models, $3 \times 3$ ) matrix of equations need to be solved during the iteration process.

After some manipulations, the viscoplastic rate equations, i.e. (1)-(4) and $(7)$, can be written in the following symbolic form $[6,7]$

$$
\dot{y}+\dot{\mathbf{X}}: \underset{z}{y}=\mathbf{H}: \dot{g}
$$

where $\dot{\mathrm{X}}$ and $\mathrm{H}$ are diagonal matrices and each contains only 2 or 3 distinct entries. The matrix $\mathbf{H}$ may be a function of time [10]. Within a typical time step $[t, t+\Delta t]$, one can obtain the analytical solution of the above equation, i.e.

$$
\underset{\sim}{y}(t+\Delta t)=\underset{\sim}{y}(t) e^{-\Delta \mathbf{X}}+\int_{\xi=t}^{t+\Delta t} e^{-\int_{\xi}^{t} \dot{\mathbf{X}} d \tau} \mathbf{H}(\xi) \underset{\sim}{g}(\xi) d \xi
$$

where

$$
\Delta \mathbf{X}=\mathbf{X}(t+\Delta t)-\mathbf{X}(t) \approx \dot{\mathbf{X}} \cdot \Delta t
$$

Here $y(t)$ is the initial value of the appropriate stresses and strains defined at the bégining of the current step. The function $g$ is defined for a specific material and is, in general, a function of the current state and the appropriate rates.

We denote the integral expression on the right hand side of $(27)$ by $\underset{\sim}{I}(\Delta t)$ so that

$$
\underset{\sim}{y}(t+\Delta t)=e^{-\Delta \mathbf{X}} \cdot \underset{\sim}{y}(t)+\underset{\sim}{I}(\Delta t)
$$

and

$$
\underset{\sim}{I}(\Delta t)=\int_{\xi=t}^{t+\Delta t} e^{-[\mathbf{X}(t+\Delta t)-\mathbf{X}(\xi)]} \mathbf{H}(\xi) \frac{\partial g}{\partial \xi}(\xi) d \xi
$$

It is seen from the above, in order to solve for $y(t+\Delta t)$ the integral $I(\Delta t)$ has to be evaluated, and the entries in $\mathrm{X}$ and in $g$ still remain unknown.

Approximation of Integral:

If the solution is exponentially decaying, it is possible to approximate the integrand in (30) by an infinite series expression 


$$
I(\Delta t)=\int_{0}^{\Delta t} e^{-\alpha z}\left[\sum_{0}^{\infty} A_{n} z^{n}\right] d z
$$

Thus, by retaining the first two powers of " $z$ ", we obtain the following approximate expression for the $i^{- \text {th }}$ component of the integral

$$
I_{i}(\Delta t)=H_{i} \Delta \Delta g_{i} \frac{1-e^{-\dot{X}_{i} \Delta t}}{\dot{X}_{i} \Delta t}+\dot{H}_{i} \dot{g}_{i}(\Delta t)^{2} \frac{e^{-\dot{X}_{i} \Delta t}-\frac{1-e^{-\dot{x}_{i} \Delta t}}{\dot{X}_{i} \Delta t}}{\dot{X}_{i} \Delta t} \text {, no sum over } \mathrm{i}
$$

where the derivatives of $g$ are approximated using the known values of this vector at the begining and at the end of the current step:

$$
\dot{g}_{i} \approx \frac{g_{i}(t+\Delta t)-g_{i}(t)}{\Delta t}
$$

Equation (32) is similar to the one derived in [6-8]. Here it incorporates the time dependency of the matrix $\mathbf{H}[10]$.

With the numerical equations outlined in the general form, it is useful to list the specific relations for the physical quantities, i.e. back stress, effective stress and inelastic strain.

Back stress:

$$
\underset{\sim}{a}(t+\Delta t)=\exp ^{-\Delta \mathrm{X}^{a}} \underset{\sim}{a}(t)+\underline{I}^{a}(\Delta t)
$$

Effective stress:

$$
\underset{\sim}{\Sigma}(t+\Delta t)=\exp ^{-\Delta \mathbf{X}^{\Sigma}} \Sigma(t)+{\underset{\sim}{I}}^{\Sigma}(\Delta t)
$$

Inelastic strain:

$$
\stackrel{\Sigma}{\varepsilon}^{I}=\underline{e}(t+\Delta t)-\frac{\sum(t+\Delta t)+a(t+\Delta t)}{2 \mu}
$$

where $\underset{\sim}{e}$ is the deviatoric strain tensor.

\section{Evaluation of Matrix $\Delta \mathrm{X}$ :}


The entries of diagonal matrix $\Delta \mathrm{X}$ appeared in (26) are related to the second invariants of inelastic deformations, which are generally unknown. These quantities are determined by an iterative process outlined as follows.

First of all, we recognize that the matrix $\Delta \mathrm{X}$ must be semipositive, i.e.

$$
\Delta \mathbf{X} \geq 0 .
$$

Since $\Delta \mathbf{X}$ is a diagonal matrix, for convenience it can be treated as an equivalent vector in its diagonal form. From (7), the general form of $\Delta \mathbf{X}$ may be written as

$$
\Delta \mathbf{X}=\mathbf{R}[y(t+\Delta t)] \Delta t
$$

This vector equation defines an implicit nonlinear system which can be solved by an iterative procedure. To this end, we define a residual vectorial function $F$

$$
\mathbf{F}=\Delta \mathbf{X}-\mathbf{R}(y) \Delta t
$$

The above equation is solved by a Newton-Raphson iterative procedure for the incremental change of $\Delta \mathrm{X}$, which is denoted by $\Delta \Delta \mathrm{X}$. Invoking Taylor series expansion and ignoring higher order terms, we find

$$
\left[\mathbf{I}-\left(\frac{\partial \mathbf{R}}{\partial \Delta \mathbf{X}}\right)^{(i)}\right] \cdot \Delta \Delta \mathbf{X}=-\mathbf{F}^{(i)}
$$

where $\Delta \Delta \mathrm{X}$ represents an error vector between two successive iterations. The updated value for $\Delta \mathrm{X}$ is thus given by

$$
\Delta \mathrm{X}^{(i+1)}=\Delta \mathrm{X}^{(i)}+\Delta \Delta \mathrm{X}
$$

Iterations are terminated when the following convergence criterion is satisfied

$$
\frac{\|\Delta \Delta \mathbf{X}\|_{2}}{\|\Delta \mathbf{X}\|_{2}} \leq \xi
$$

where $\xi$ is a tolerance limit and $\|\cdot\|_{2}$ designates a Euclidean norm. It is noted again that $(40)$ represents a system of $2 \times 2$ simultaneous equations regardless whether for a two-dimensional or three-dimensional problems. This is obviously a major advantage of the asymptotic integration algorithm over 
the trapezoidal rule where one has to solve a system of $19 \times 19$ simultaneous equations for every iteration.

In (40), the coeffficient matrix, denoted by $J=\frac{\theta R}{\partial \Delta X}$ represents a $2 \times 2$ Jacobian matrix, which may be derived analytically or defined by numerical means. To improve computational efficiency of the procedure, we have obtained the analytical expressions for both Walker's and Robinson's models.

\subsection{Time Step Control}

As mentioned in the previous susections, numerical stability of trapezoidal and asymptotic integration algorithms is assured by the fact that both procedures are implicit methods. However, special care must be given to insure solution uniqueness, or the invertibility of equation (40). To this end, a Lipschitz condition $[3,5,18]$ is employed. The Lipschitz number is defined by:

$$
L=\sup _{\xi \in \Delta t}\|\mathbf{J}(\xi)\|_{p}
$$

where \|\|$_{p}$ is the $p^{\text {th }}$ order norm.

Lipschitz theorem states that if $0 \leq L<, 1$ then there exists a unique solution and the iterates $\Delta \mathbf{X}^{i}$ will converge after sufficient number of itarations is performed. This theorem defines a convergence condition for the Jacobian matrix, i.e.

$$
\max _{i}\left|\lambda_{i}(\mathbf{J})\right| \leq\|\mathbf{J}\|_{p}<1
$$

where $\lambda_{i}$ is the $i$-th eigenvalue of $J$. The requirement in (44) insures the existence of the inverse of $(I-J)$. The particular p-norm is at the discretion of the user. Fulfilling this condition, the algorithm is unconditionally stable and will yield a unique solution.

Our time step control makes use of the Lipschitz number. That is, during the analysis the magnitude of the Jacobian matrix is being monitored. Whenever its norm exceedes an allowable limit, the global time step is subdivided by the calculated Lipschitz number. Thus, the subincrement step size is given by

$$
\Delta \tau=\frac{\Delta t}{L(t)}
$$


where $L(t)$ is the current Lipschitz number and $\Delta t$ is the global time step.

\section{Computer Implementation}

The viscoplastic models discussed in the previous sections have been written in the form of a material module for finite element computations. Although the module was written for implementation into a specific code, i.e. NFAP [16], it is adaptable to other general purpose finite element codes with minimum effort.

Basically, the material module constitutes two main branches: integration methods and material functions. In the integration branch, one has the option to choose explicit schemes, e.g. forward Euler or Runge-Kutta methods, or implicit schemes, such as the trapezoidal rule or the asymptotic integration method. In the material function branch, one may select Walker [10], Robinson [11] or any other viscoplastic model. The coding is transparent enough so that user may easily add another integration method and/or viscoplastic model.

The material data are calculated at all integration (or Gauss) points of all elements: that is, all continuum elements $(2 \mathrm{D}, 3 \mathrm{D}$, plate or shell element) share the same material module. The material module is called by the main program in three stages for each global time step: 1) element stiffness calculation, 2) calculation of the internal forces for global equilibrium check (iterations), and 3) stress and strain output. Data transfer between the main program and the material module is achieved through the subroutine argument list and common blocks. In entering the material module, the following data are defined: ${\underset{\sim}{n}}_{n},{\underset{\varepsilon}{n}}_{n}^{I},{\underset{\sim}{a}}_{n}, k_{n}, g_{n}, T_{n}, t_{n}, \Delta t$, and ${\underset{\varepsilon}{n+1}}_{n+1}, T_{n+1}$, where ()$_{n}$ indicates quantity at time $t ;()_{n+1}$, quantity at time $[t+\Delta t]$. When calculations are done in the material subroutines, the material stiffness matrix and the updated inelastic strains, stresses and state variables corresponding to the current deformation are passed onto the main program. A skeletal block diagram for the material module outlining the major calculation controls are shown in fig.(1) and specific functions of each control routine are described below.

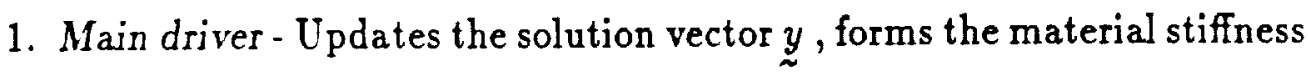


matrix $\mathbf{D}(t+\Delta t)$, and if necessary, adjusts the global time increment $\Delta t$ to obtain the local subincrement $\Delta \tau$ at each Gauss point.

The main driver calls upon two control routines: numerical algorithm control and subincremental time control.

2. Numerical algorithm control - At each time step, it selects the appropriate algorithm optioned by the analyst, and computes the corresponding iteration vector $y_{n+1}^{i}$ with a suitable convergence test.

3. Material function control - Calculates the inelastic strain and associated evolution equations for a specific viscoplastic model. If the asymptotic algorithm is employed, it evaluates the coefficient matrices $X, H$ and the vector $g$ defined in (26) for either Walker's or Robinson's model.

4. Jacobian matrix control - For implicit algorithms, it evaluates the Jacabian matrix of the material model and checks a criterion for solution convergence and invertibility of the matrix equations involved.

5. Subincremental time control - Using the criterion defined in the previous section, it determines whether or not the global time step $\Delta t$ is to be divided into subincrements: $\Delta \tau=\Delta t / L$, where $\mathrm{L}$ is the Lipschitz number, see (45).

\section{Numerical Examples}

Three sample problems are included to demonstrate the utility of numerical algorithms implemented for finite element applications of viscoplastic structures. Although the constitutive equations incorporated in the program can be utilized for any general three-dimensional state of stress, the problems considered here are merely two-dimensional. In our analysis, some numerical difficulty was encountered due to the mathematical discontinuity existed in Robinson's constitutive equations and ways to alleviate the aforementioned difficulty are discussed. Further, a comparison of the numerical performance, in terms of CPU time and solution accuracy, between the trapezoidal rule and aymptotic algorithm is also included in the present study. 


\subsection{A Thick Walled Cylinder}

The first example is a thick walled cylinder subjected to a time dependent internal pressure, $p(t)$, which undergoes a full loading- unloading cycle. The finite element model contains 12 axi-symmetric elements and is shown in fig.(2) along with the input pressure function. The material for the cylinder is represented by Walker's isotropic model, which does not have any dicontinuities in its mathematical formulation. The main objective of solving this problem is to verify the coding for the various numerical integration procedures considered.

Material constants for this problem are [6]:

$$
\begin{aligned}
& \mathrm{T}=1600 \mathrm{~F} \quad \mathrm{E}=18.6 \cdot 10^{3} \mathrm{Ksi} \quad k_{0}=91505 \mathrm{Ksi} \quad \nu=0.345 \\
& \mathrm{p}=3 . \quad \mathrm{n}=5.128 \quad n_{1}=1.158 \quad n_{2}=0 . \\
& \begin{array}{llll}
n_{3}=5 \cdot 10^{6} & n_{4}=672.6 & n_{5}=0 & n_{6}=0
\end{array} \\
& n_{7}=8.98 \cdot 10^{-4} \quad n_{8}=0 \quad n_{9}=0
\end{aligned}
$$

To carry out the incremental analysis, the entire load cycle is divided into 50 time steps with variable step sizes within the four quarters of the load cycle. Solution was obtained by using four different integration schemes: the asymptotic algorithm, the trapezoidal rule with Newton-Raphson iterations, the forward Euler and Runge-Kutta procedures with error checks $[3,9,12,18]$. The resulting hoop stresses are plotted in figs.(3) and (4). As seen in the plots, no visible difference can be detected among the numerical results obtained by the different integration schemes.

The CPU time for each of the integration schemes is listed in Table 1. It appears that both the Runge-Kutta method and the asymptotic algorithm consume about $20 \%$ less CPU time than the implicit schemes. The Euler method seems to be the most time-consuming algorithm in order to achieve the same degree of solution accuracy.

\subsection{A Simply Supported Beam}

The second example is a simply-supported beam under a state of plane stress. The loading function is a full-cycle displacement prescribed at the center of the beam. The finite element model and the applied load function are plotted in fig.(5). For this problem, Robinson's isotropic model is employed. As noted before, Robinson's model contains mathematical discontinuities which 
may pose numerical diffculty during the course of integrating the constitutive equations. We use this problem to illustrate how numerical difficulty may arise as a result of discontinuities existing in the constitutive equations. To remove such difficulty, we need to monitor the variation of the Jacobian matrix so that its norm must be kept within a certain allowable tolerance. Otherwise, numerical anomaly will occur, which in turn causes either erroneous or non-unique solutions.

The material constants used for this example are[10]:

$$
\begin{array}{llll}
\mathrm{T}=1000.4 \mathrm{~F} & \mathrm{E}=22,480 \mathrm{Ksi} & k_{T}=0.82 \mathrm{Ksi} & \nu=0.345 \\
\mathrm{n}=4 & \mathrm{r}=3.61 \cdot 10^{7} & \mathrm{~m}=7.73 & \beta=1.5 \\
\mathrm{R}=9 \cdot 10^{-8} & \mathrm{H}=1.37 \cdot 10^{4} & G_{0}=0.1 &
\end{array}
$$

Regions of Numerical Difficulties - When solving the beam problem using Robinson's model with the asymptotic algorithm, oscillations in the numerical solution were detected. There are in fact two different types of numerical oscillations: case 1 ) $\|\mathbf{J}\| \geq 1$, and case 2 ) $\|\mathbf{J}\| \approx 0$. The first case occurs when the rate of change of the state variables is very rapid (or high rates are encountered), thus the corresponding constitutive rate equations are in a stiff regime. The second case represents another extreme; that is, the material is almost responding elastically with very little inelastic deformation. Thus, solution of (26) leads to numerical drifting.

To further illustrate the numerical phenomenon stated above, we have plotted in fig.(6) the bending stress vs. the back stress at an integration point near the upper fiber near the center of the beam. As seen in the figure, the back stress is changing much faster then the corresponding stress component, thus leading to singularity of the coefficient matrix in (26). This phenomenon is further verified from the plots of the Jacobian norm vs. the number of local iterations at the integration point in fig.(7). The end result is that a considerable amount of CPU time is consumed for very little improvement in solution accuracy. In fig.(8), the dots close to the abscissa represent the drifting of the Jacobian matrix. To alleviate such difficulty, we take an average value of $\Delta \mathrm{X}$ in (40) evaluated between two successive iterations. By doing this, the numerical drift was immediately elimianated.

To stabilize the numerical oscillation of the first case, a step control procedure was implemented that checks the magnitude of Jacobian norm. When 
the norm of the coefficient matrix in (40), $\|\mathbf{I}-\mathbf{J}\|$ is kept within a tolerance limit, oscilations in the numerical solution of (26) are dramatically reduced to relatively stable values as indicated in figs.(9). It shows a reduction in the Jacobian norm when the integration steps are limited within a reasonable value. As a result, the integration algorithm yields a unique convergent solution.

The same problem was also analyzed for the trapezoidal rule and RungeKutta method with error control [17]. In the case of trapezoidal rule, a constant fixed-step history was employed, whereas an adaptive step procedure was activated in the case of asymptotic algorithm. The trapezoidal rule failed to yield a convergent solution in the critical discontinuity region as indicated in fig.(10). On the other hand, the solutions obtained from the asymptotic algorithm and the Runge-Kutta method converged quite nicely, since both methods have a built-in step control.

The CPU time for the three integration methods is detailed in Table (2). The time required by the asymptotic algorithm is about $50 \%$ less than that of the trapezoidal rule. For a larger size problem, the time saving will be much more significant.

\subsection{A Cylindrical Thrust Chamber}

The third example is a cross section of a cylindrical rocket thrust chamber which is subjected to thermal as well as pressure cycling [19]. Due to the repetitous pattern of its cross section, only one sector including the cooling channel is considered for finite element analysis, fig. (11). In addition to the thermal loading, the chamber is subjected to a time dependent internal pressure; $p_{1}(t)$ acting on the cooling channel surface, and $p_{2}(t)$ acting on its inner surface. The temperature and pressure functions $p_{1}$ and $p_{2}$ follow the same time history and the corresponding function for one typical loading cycle is shown in fig.(12). The sector of the chamber is modeled by 348 -node plane strain elements, fig.(12). The chamber material is assumed to follow Robinson's isotropic model with the same material constants as were used for the beam. In the finite element analysis, both the asymptotic algorithm and trapezoidal rule were used for comparison of solution efficiency.

Shown in fig.(13)is the deformed shape of the thrust chamber cross section at the end of one loading cycle. From the analysis, considerable inelastic strain accumulations occurred at the inner corner of the cooling channel, 
which in turn caused bowing effect near the central section. This is called the "dog house" effect and it is observed in experiments [20]. Also shown in fig.(14) is the maximum inelastic strain history that occurred at the corner of the cooling channel.

We use this problem to compare the solution efficiency between the asymptotic algorithm and trapezoidal rule. To this end, we employ two different step histories: 1) a constant step history for both methods as a basis of comparison, and 2) a variable step history with step control for the asymptotic algorithm. After several trials, it was found that 1000 constant, global, time step increments were needed to obtain a convergent solution for the trapezoidal integration rule. Table (3) summarizes the CPU time ratios between the two methods. The CPU time ratio per iteration is defined as

$$
C P U \text { Ratio }=\frac{C P U \text { time using trapezoidal rule }}{C P U \text { time using asymptotic algorithm }}
$$

As seen in the table, the ratios vary somewhat during the course of the analysis, i.e. within the range of $1-5$. It is pointed out that the asymptotic algorithm is particularly efficient when the rate of change of the state variables becomes large. In addition to the constant, step history, we employed the asymptotic algorithm with an adaptive step control. In this case, the method becomes even more attractive since it requires only about one-fourth of the CPU time consumed by the trapezoidal method.

\section{Conclusion}

Two state variable-based viscoplastic models, namely Walker's and Robinson's models, have been implemented into a general purpose finite element code for structural applications of metals deformed at elevated temperatures. These models are represented in a material module form so that they may be easily modified or transplanted to other finite element codes. The addition of new viscoplastic models or integration routines is relatively transparent to an investigator. A comparative study has been made on the numerical performance of two implicit integration methods: the trapezoidal rule and the asymptotic algorithm. It was found that the asymptotic algorithm becomes very effective if a self adaptive scheme based on an error check of the 
Jacobian matrix and subincremental step control is utilized at the material's integration points.

\section{Aknowledgement}

Research work reported in this paper is supported by NASA Lewis Research Center, Cleveland, Ohio, under a grant: NAG 3-901. Technical Officer of this project is Dr. Steven M. Arnold. We would also like to thank Dr. Alan D. Freed of NASA Lewis Research Center for his invaluable comments and suggestions about this paper. 


\section{References}

1. Willam, K. J., Numerical Solution of Inelastic Rate Process, Comp. and Struc., Vol. 8, 1978, pp. 511-531

2. Chaboche, J. L., A Review of Computational Methods for Cyclic Plasticity and Viscoplasticity, Computational Plasticity, Ed. by R. J. Owen, E. Hinton and E. Onate, Proc. of Int. Conference on Computational Plasticity, Barcelona, Spain, April 6-10, 1987.

3. Lambert, J. D., Computational Methods in Ordinary Differential Equations, John Wiley \& Sons, 1973.

4. Kumar, V., Marjaria, M. and Mukherjee, S., Numerical Integration of Some Stiff Constitutive Models of Inelastic Deformations, J. of Eng. Mat. and Tech., Vol. 102, 1980, pp. 92-96

5. Gear, G. W., Numerical Initial Value Problems in Ordinary Differential Equations, Prentice - Hall, Englewood Cliffs, N. J. , 1981.

6. Chulya, A. and Walker, K. P., A New Uniformly Valid Asymptotic Integration Algorithm for Elasto-Plastic-Creep and Unified Viscoplastic Theories Including Continuum Damage, NASA Lewis Research Center, TM-102344, 1989.

7. Walker, K. P. and Freed, A. D., Asymptotic Integration Algorithm for Nonhomogeneous, Nonlinear, First Order Ordinary Differential Equations, NASA TM-103793 NASA Lewis Research Center, Cleveland, $\mathrm{OH}, 1991$.

8. Freed, A. D. and Walker, K. P., Refinments in a Viscoplastic Model, NASA TM-102338, NASA Lewis Research Center, Cleveland, OH, 1989.

9. Chang, T. Y. and Chang, J. P., Viscoplastic Analysis of Metalic Structures by Finite Element Methods, a technical report submitted to NASA Lewis Research Center, Department of Civil Engineering, University of Akron, Akron, OH, 1985. 
10. Walker, K. P., Representation of Hastelloy-X Behaviour at Elevated Temperature with a Functional Theory of Viscoplasticity, paper presented at ASME/PVP Century 2 Energy Technology Conf., San Francisco, CA, August 1980.

11. Robinson, D. N., United Creep-Plasticity Constitutive Equation at Elevated Temperature, Oak Rigde National Laboratory (ORNL) TM$5969,1978$.

12. Bass, J. M. and Oden, J. T., Numerical Solution of the Evolution Equations of Damage and Rate Dependent Plasticity, Int. J. Engng. Sci., Vol. 26, No.7, 1988, pp. 713-740

13. Freed, A. D. and Chaboche, J. L., Viscoplasticity : A Thermodynamic Formulation, NASA TM-102388 NASA Lewis Research Center, Cleveland, OH., 1989.

14. Brockman, R.A., Explicit Forms for the Tangent Modulus Tensor in Viscoplastic Stress Analysis, Int. J. for Num. Meth. in Eng., Vol. 20, 1984, pp.315-319

15. Pierce, D., Shih, C. F. and Needelman, A., A Tangent Modulus Method for Rate Dependent Solids, Comp. and Struc., Vol. 18, 1987, pp.875-887

16. Chang, T. Y., A Nonlinear Finite Element Analysis Program (NFAP), Department of Civil Engineering, University of Akron, Akron, OH, 1988.

17. Chang, T. Y., Chen, J. Y. and Chu, S.C., Viscoplastic Finite Element Analysis by Automatic Subincrementing Technique, J. of Eng. Mech., $A S C E$, Vol. 111(1), 1988, pp.80-96.

18. Shampine, L. F. and Gordon, M. K., Computer Solution of Ordinary Differential Equations - The Initial Value problem, W.H. Freeman $\mathcal{E}^{6}$ Company, San Francisco, 1975.

19. Johnson, L.W. and Riess, R. D., Numerical Analysis, Addison - Wesley Publishing, Reading, Mass., 1980. 
20. Quentmeyer, R. J. , Experimental Fatigue Life Investigation of Cylindrical Thrust Chambers, NASA TM X - 73665 NASA Lewis Research Center, Cleveland, OH, 1977. 
Table 1: CPU Ratio of a Thick Walled Cylinder (Walker's Model)

\begin{tabular}{|c|c|c|c|}
\hline \hline & $\begin{array}{c}\text { Explicit } \\
\text { Runge Kutta }\end{array}$ & $\begin{array}{c}\text { Implicit } \\
\text { trap. }\end{array}$ & $\begin{array}{c}\text { Forward } \\
\text { Euler }\end{array}$ \\
\hline \hline $\begin{array}{c}\text { Cal.of } \\
\text { Monlin. } \\
\text { Mat. }\end{array}$ & 1.168 & 1.137 & 1.7 \\
\hline \hline
\end{tabular}

Table 2: CPU Ratio of a Simply Supported Beam (Robinson's Model)

\begin{tabular}{|c|c|c|c|}
\hline & $\begin{array}{c}\text { Explicit } \\
\text { Runge-Kutta } \\
\text { 25 steps }\end{array}$ & $\begin{array}{c}\text { Explicit } \\
\text { Runge-Kutta } \\
\text { 50 steps }\end{array}$ & $\begin{array}{c}\text { Implicit } \\
\text { Trap. }\end{array}$ \\
\hline \hline $\begin{array}{c}\text { Cal.of } \\
\text { Nonlin. } \\
\text { Mat. }\end{array}$ & 1.8 & 2.5 & no \\
convergence \\
\hline \hline
\end{tabular}

Table 3: CPU Ratio of a Th- Thast Chamber

\begin{tabular}{|c|c|} 
& (Robinson's Model) \\
\hline \hline Step & CPU \\
Range & Ratio \\
\hline \hline & \\
$1-2$ & 5 \\
$3-200$ & 1 \\
$201-400$ & 2 \\
$401-600$ & 2.5 \\
$601-800$ & 2 \\
$801-1000$ & 3 \\
\hline
\end{tabular}




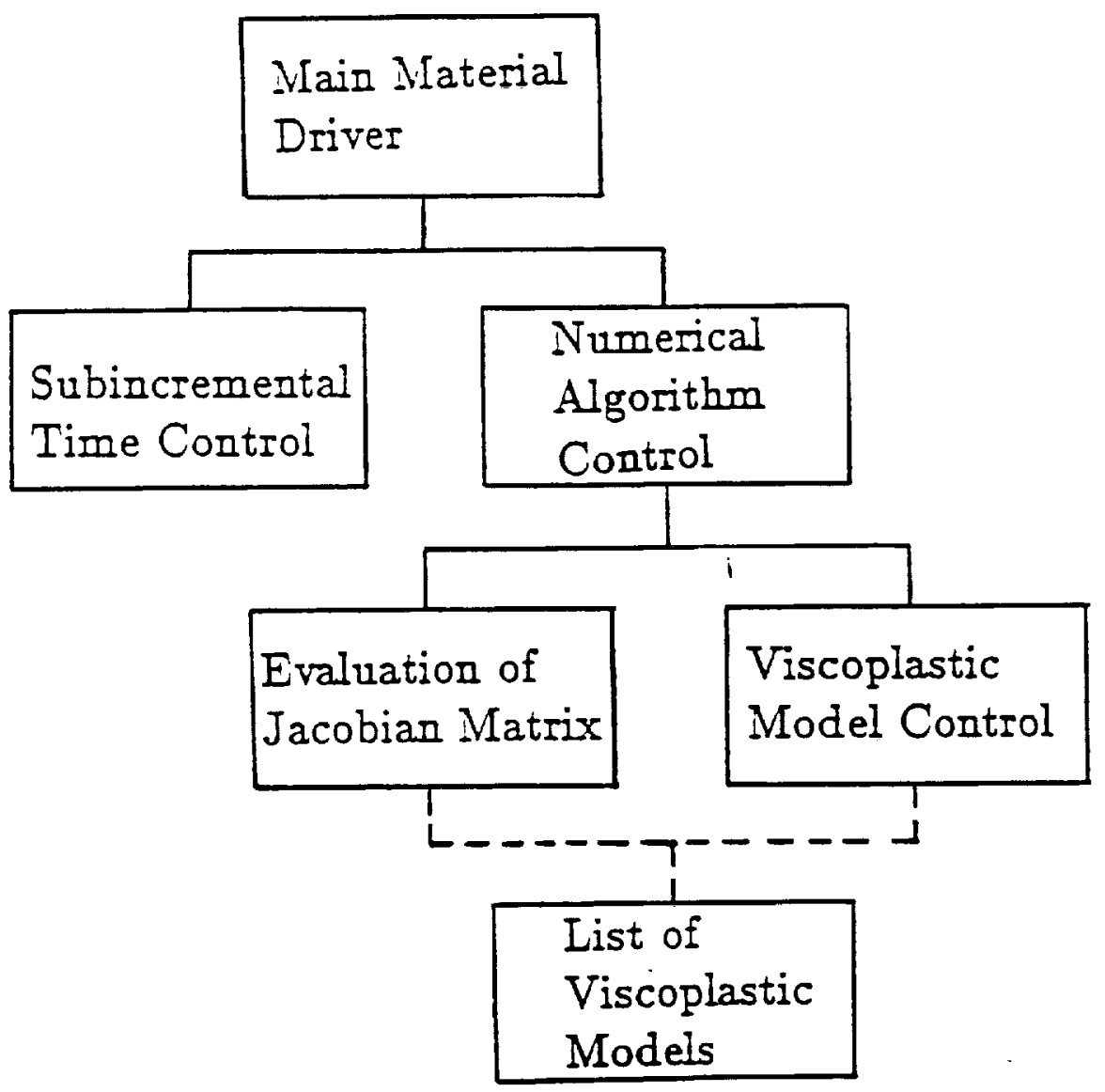

Figure 1: Block Diagram of Material Module 


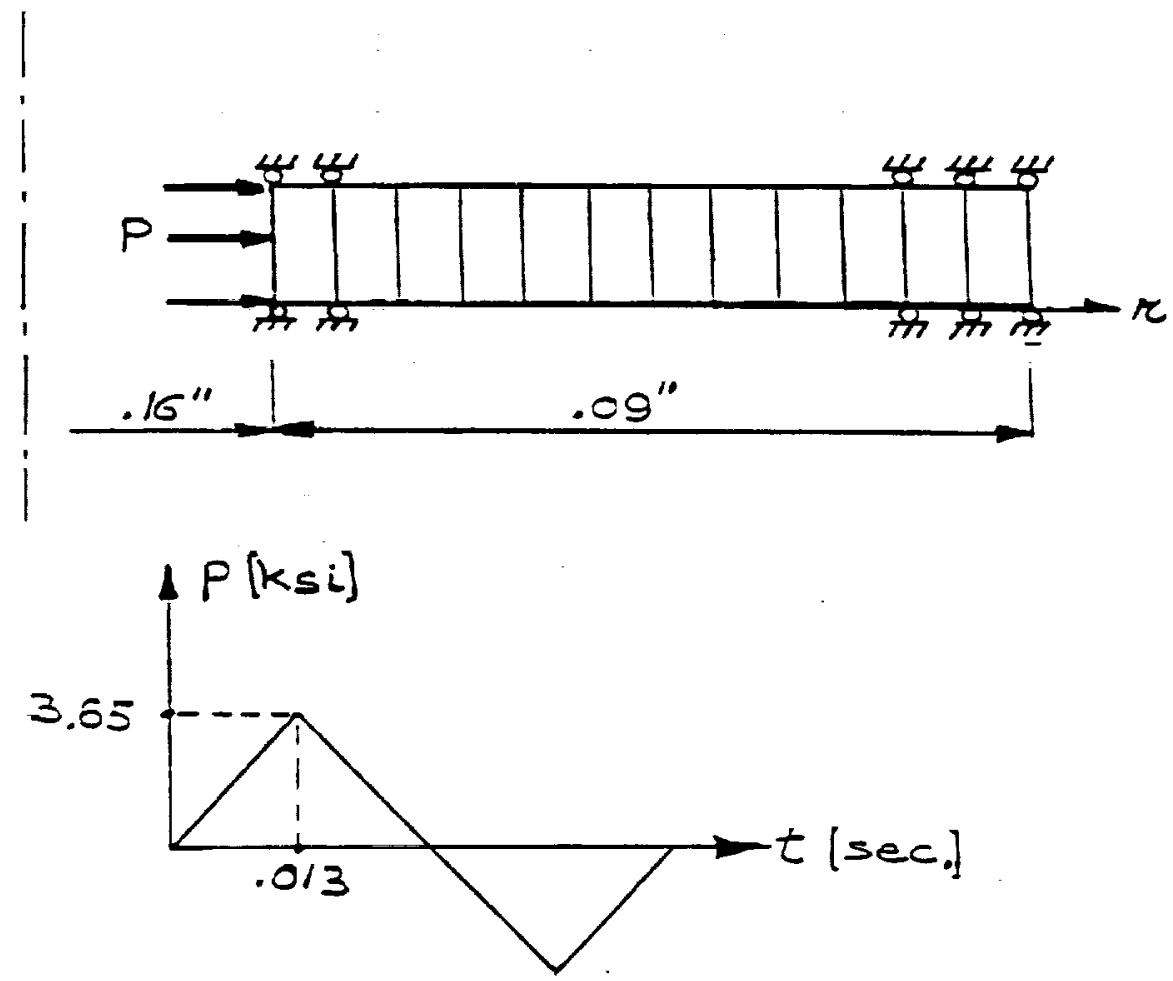

Figure 2: Thick Walled Cylinder \& Loading Pressure Function 


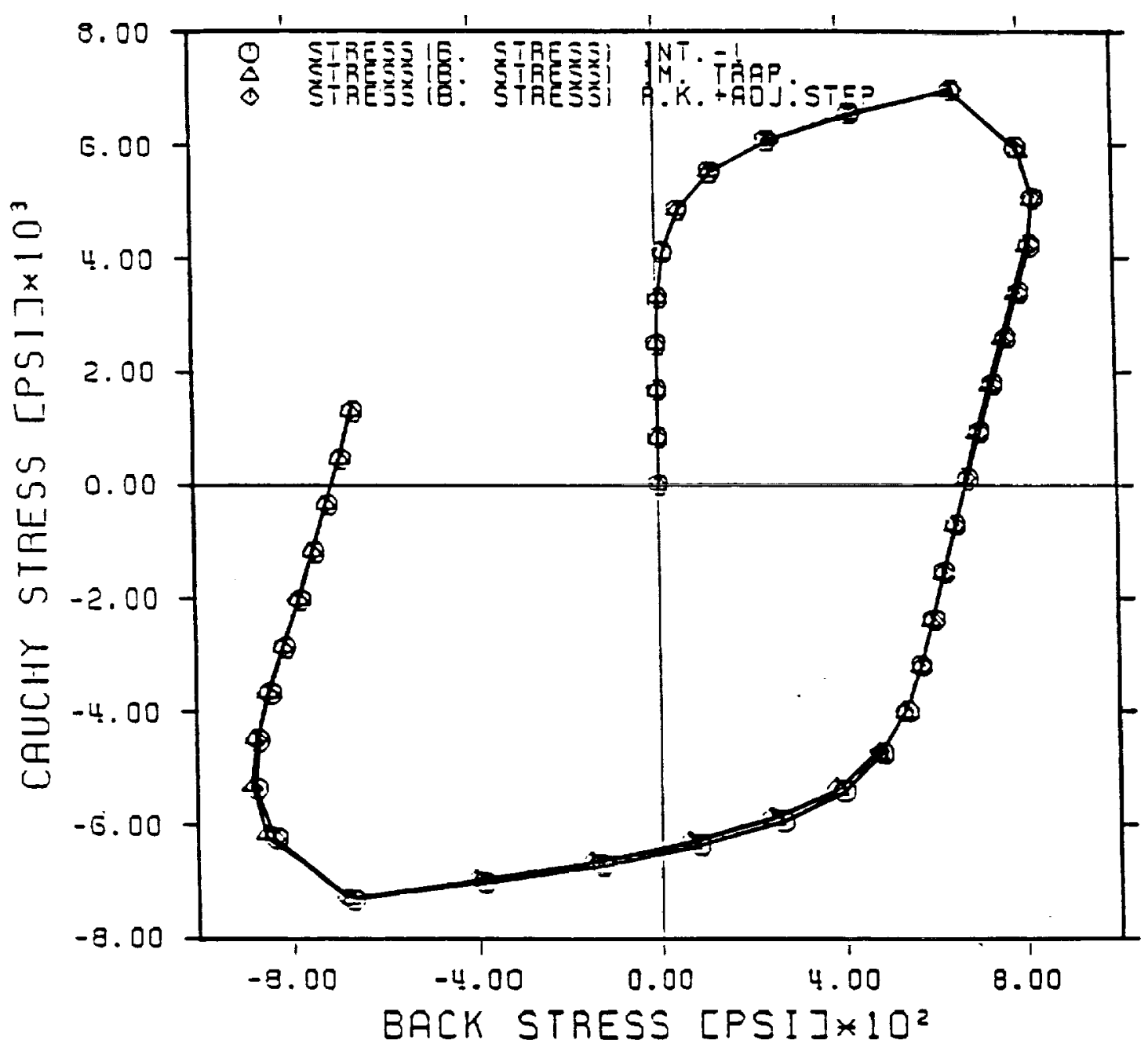

Figure 3: Walker's Isotropic Material for a Thick Walled Cylinder $\sigma_{\theta}-a_{\theta}$ 


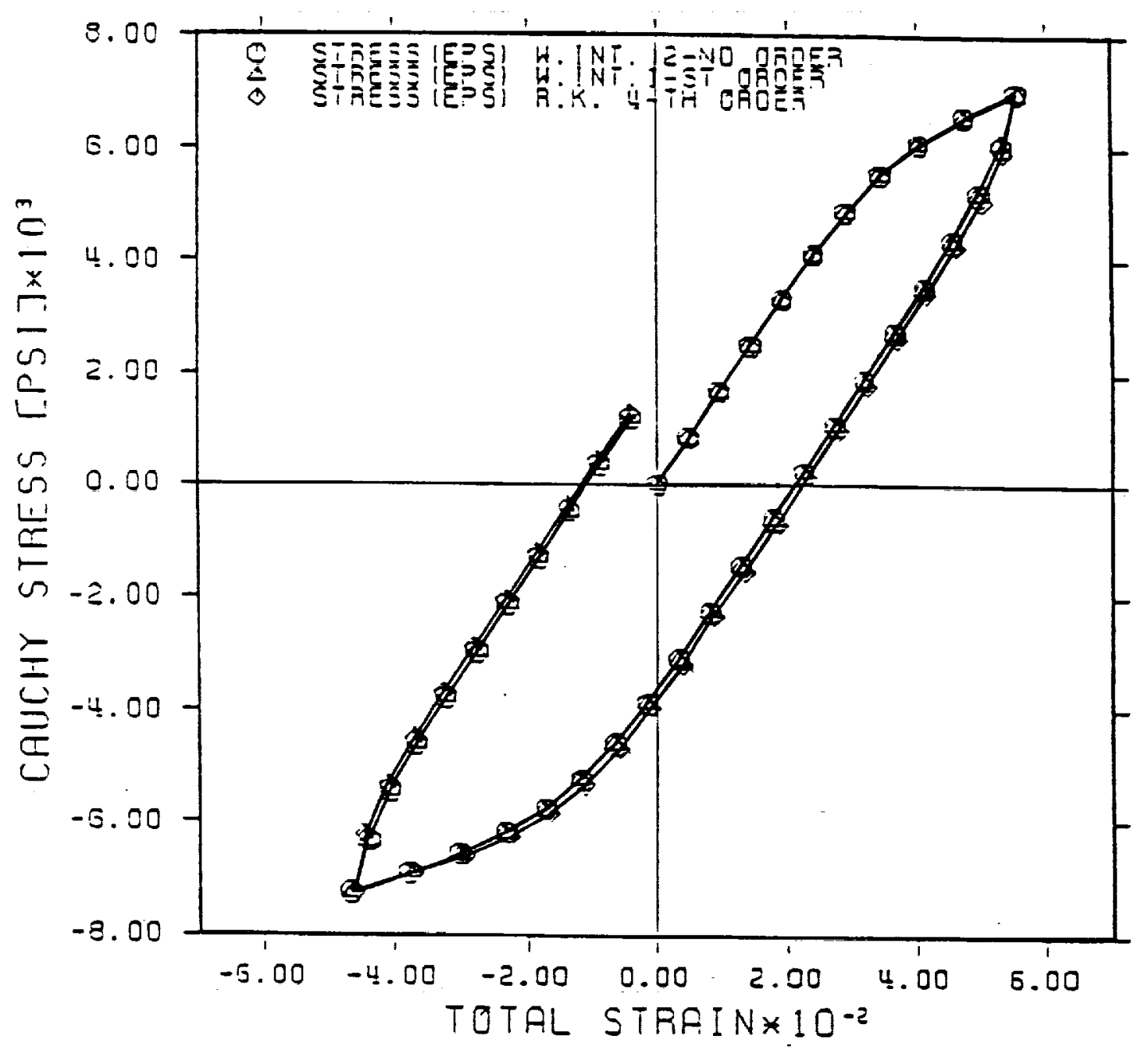

Figure 4: Walker's Isotropic Material for a Thick Walled Cylinder $\sigma_{\theta}-\epsilon_{\theta}$ 

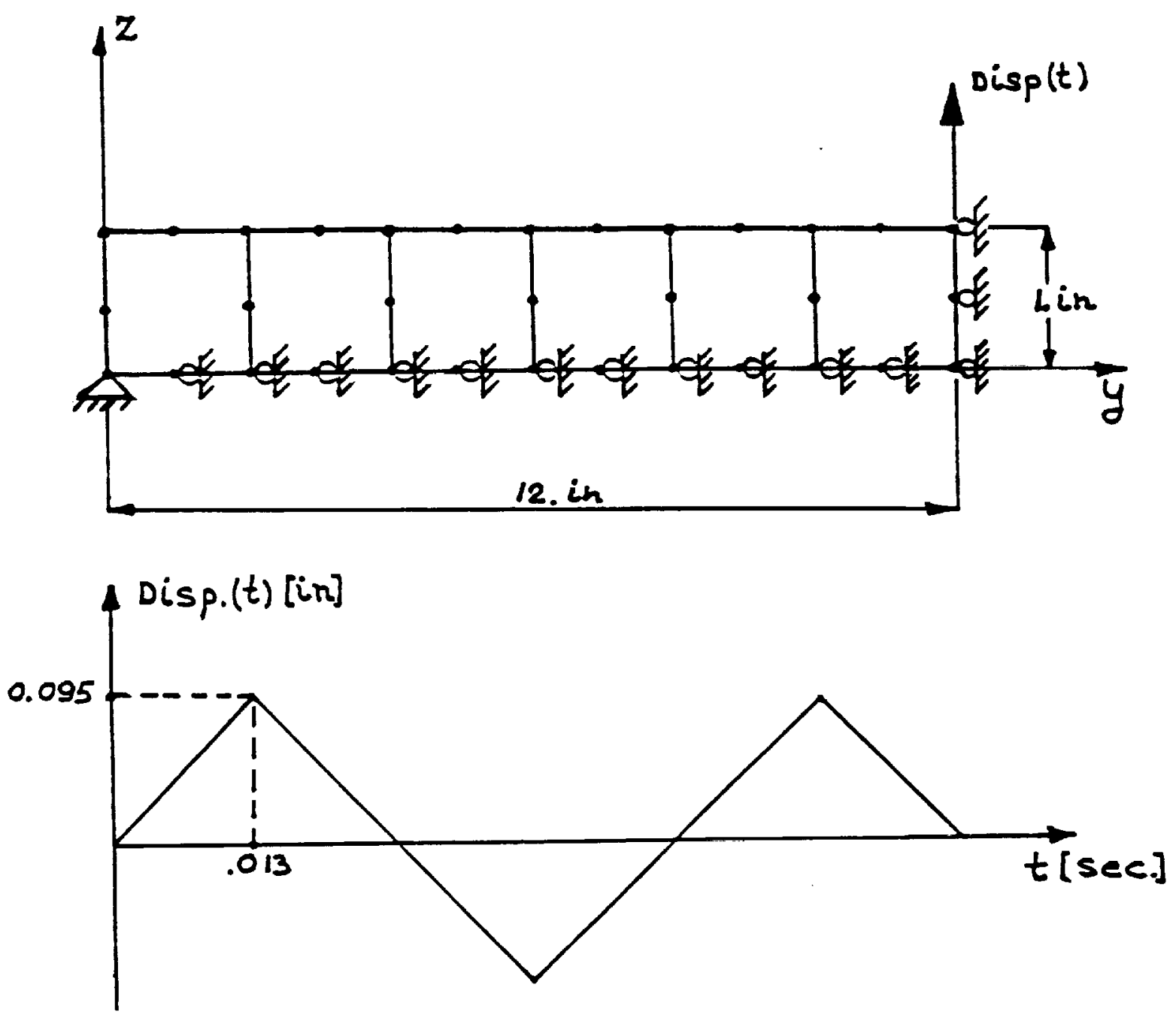

Figure 5: Simply Supported Beam \& Loading Displacement Function 


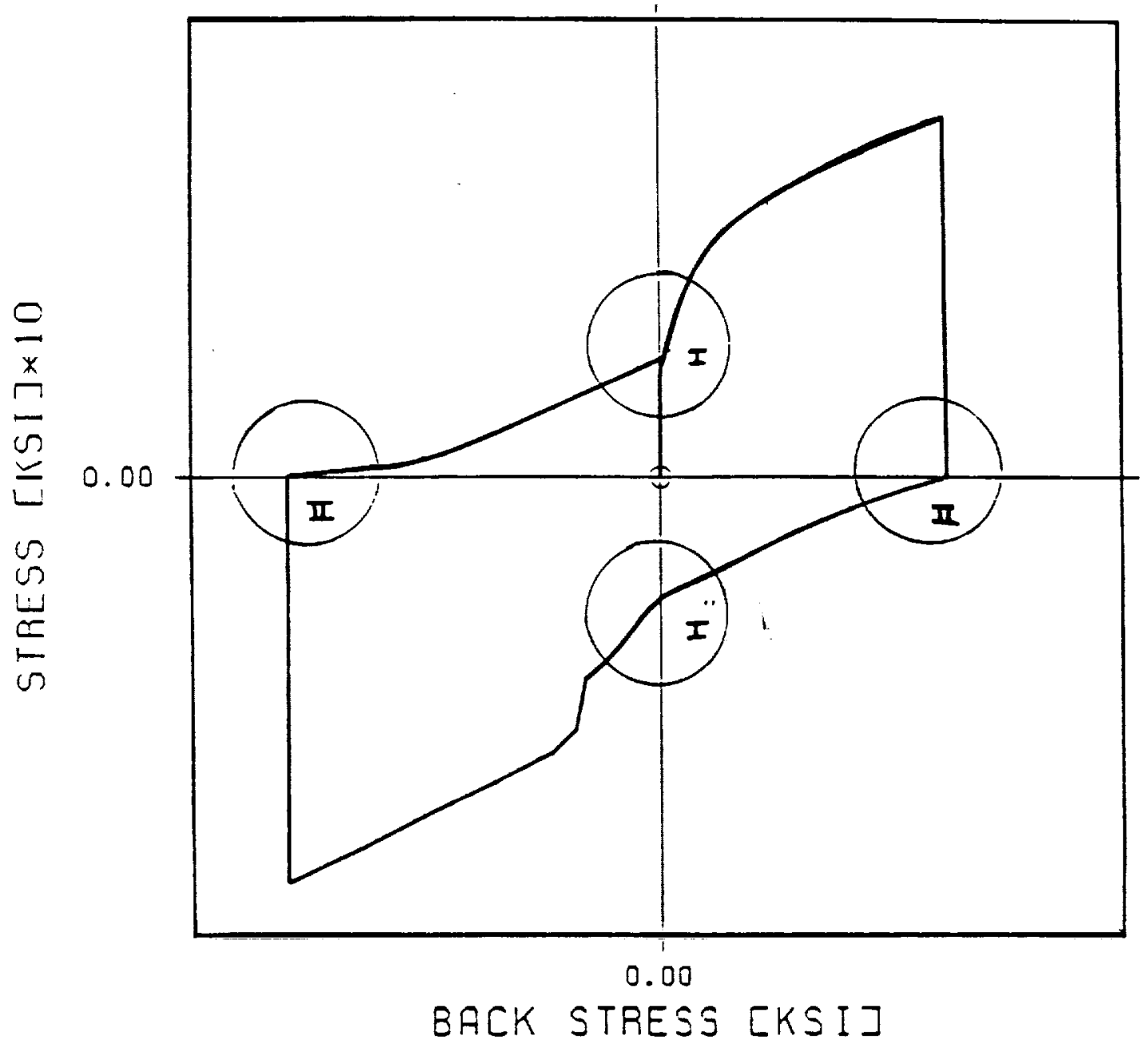

Figure 6: Robinson's Material - Zones of Numerical Difficulties 


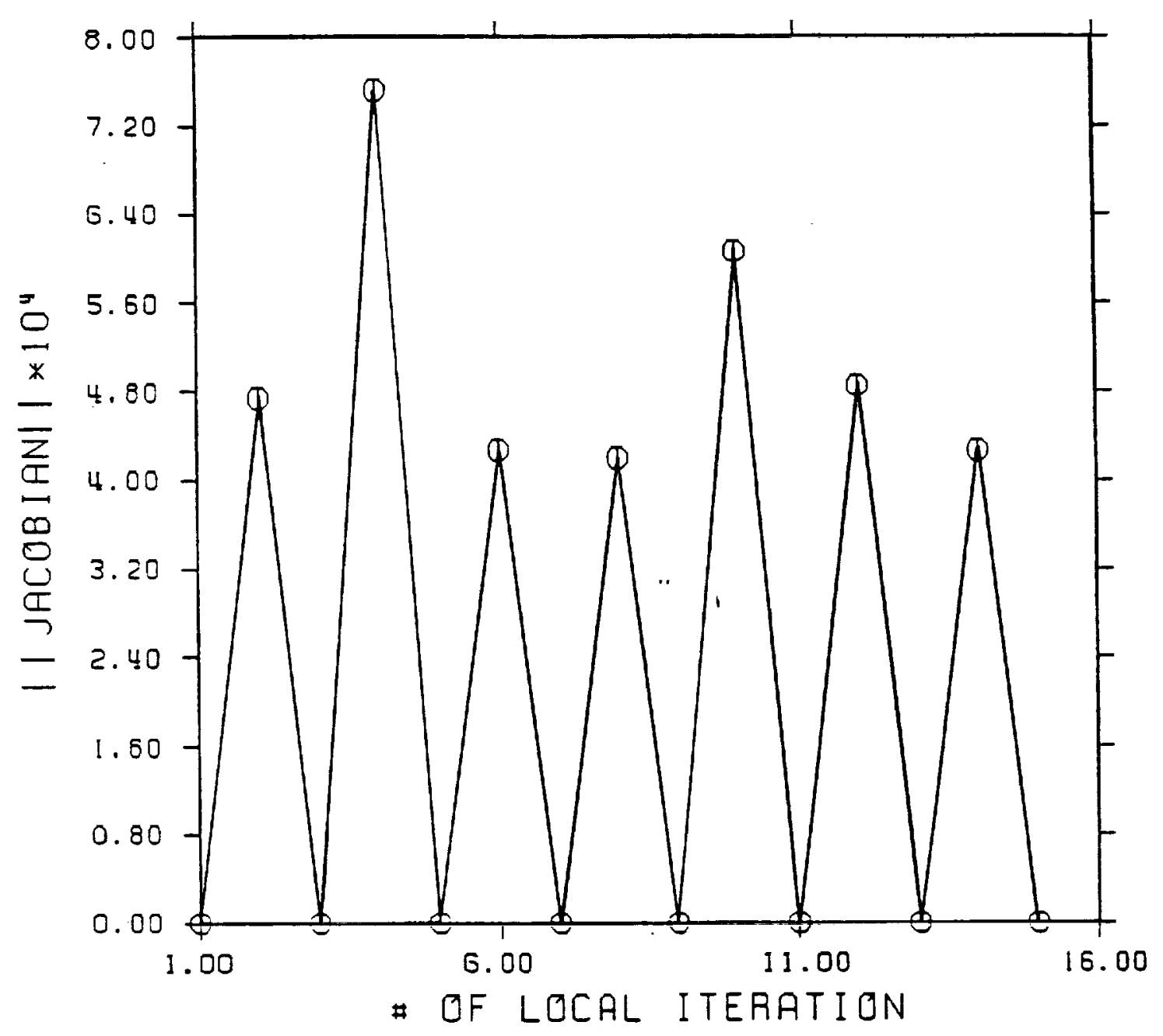

Figure 7: Jacobian Oscilations - No step control 


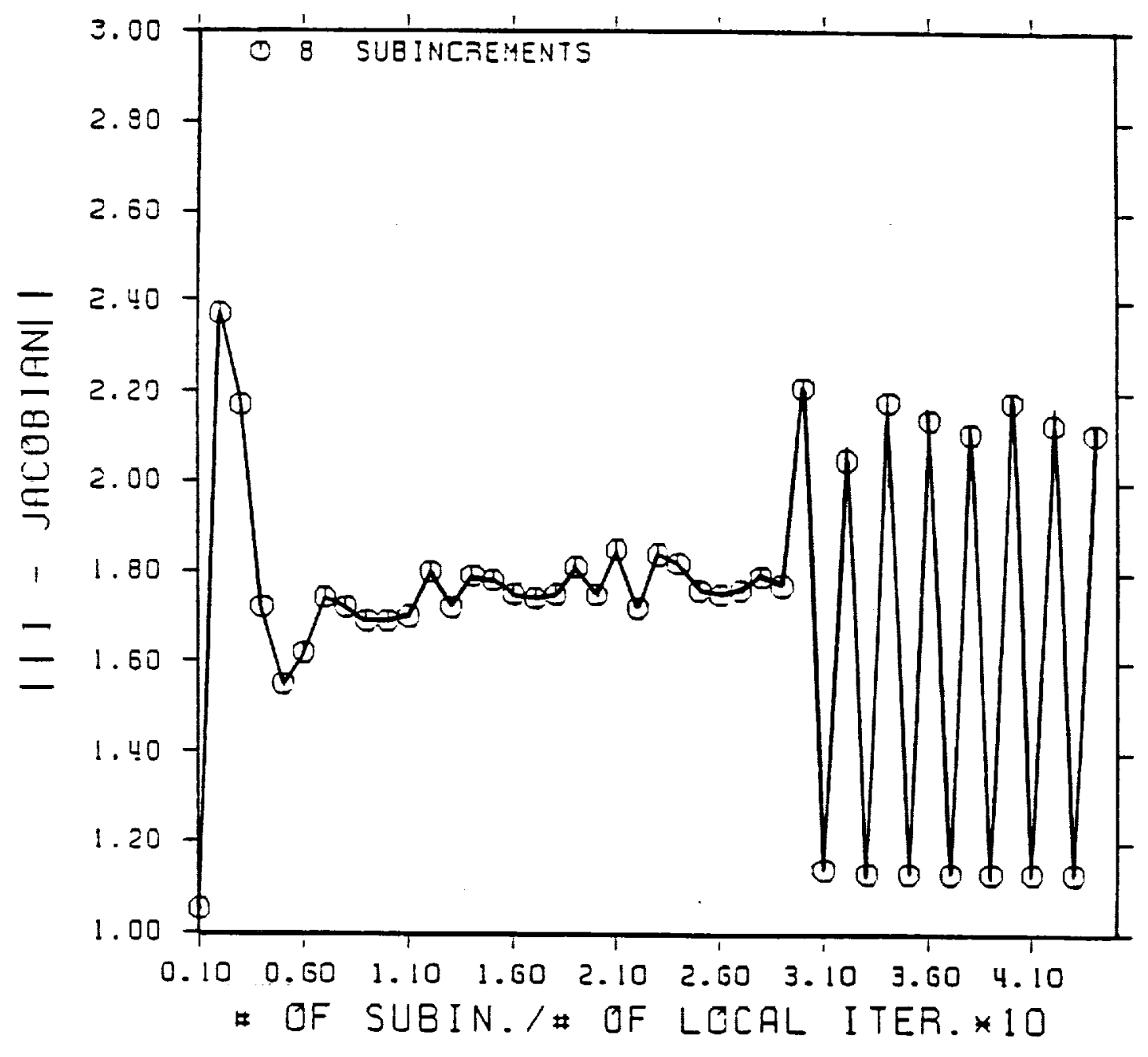

Figure 8: Jacobian Oscilations - $\|\mathbf{J}\| \approx 0$. 


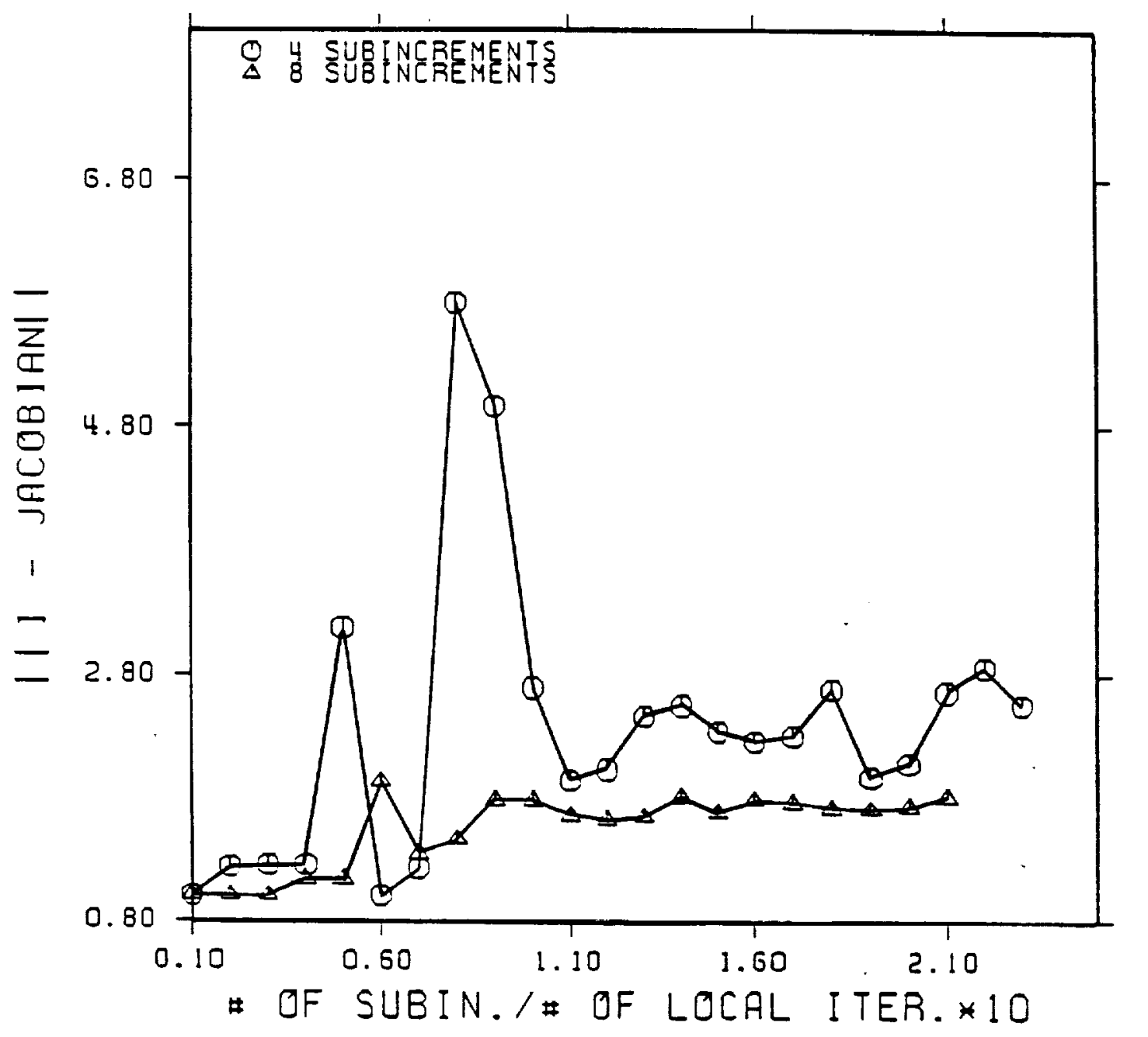

Figure 9: Jacobian Oscilations - With step control 


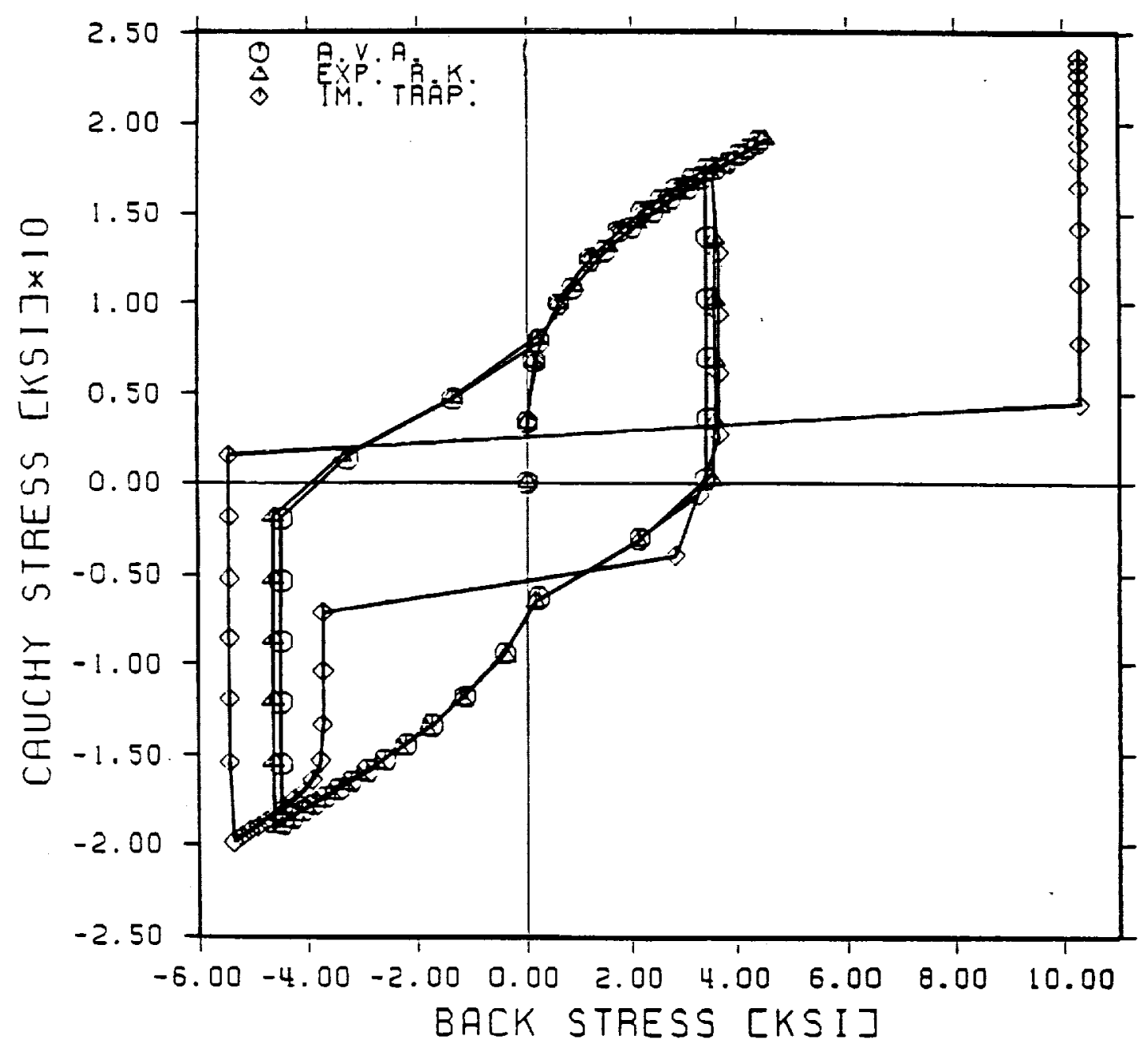

Figure 10: Robinson's Isotropic Material for a Simply Supported Beam $\sigma_{y y}-a_{y y}$ 


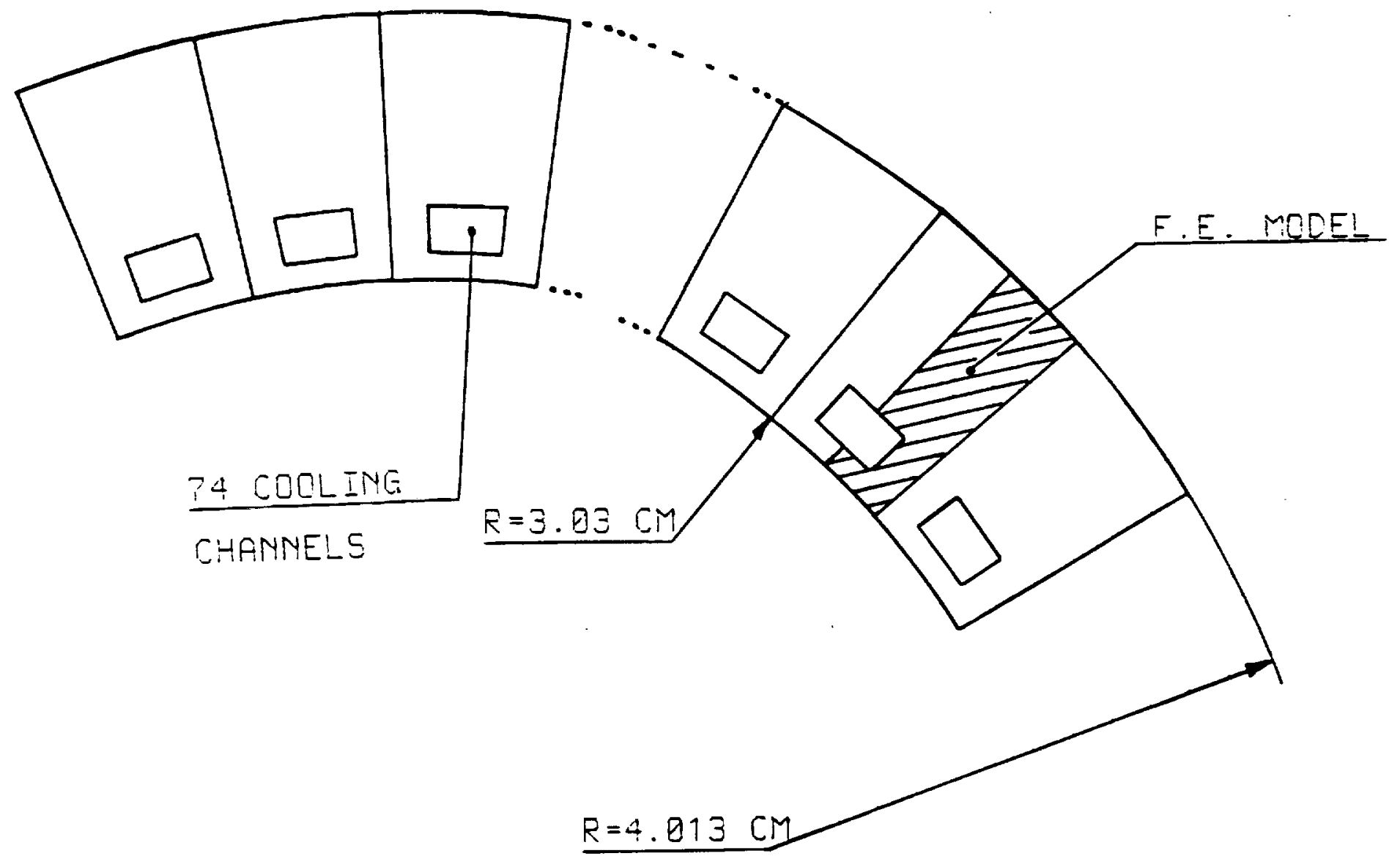

Figure 11: Cross Section of a Cylindrical Thrust Chamber 

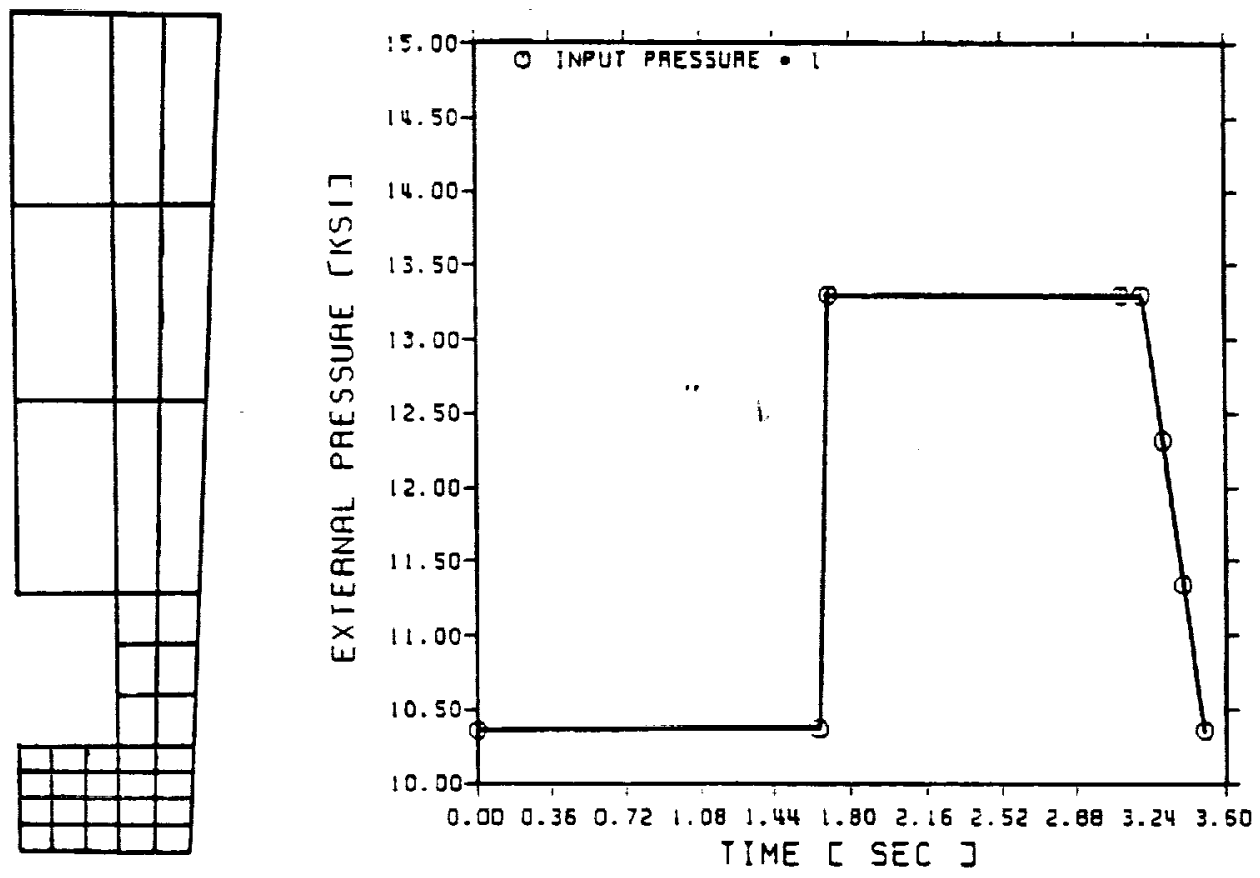

Figure 12: Thrust Chamber Model \& Pressure Function 


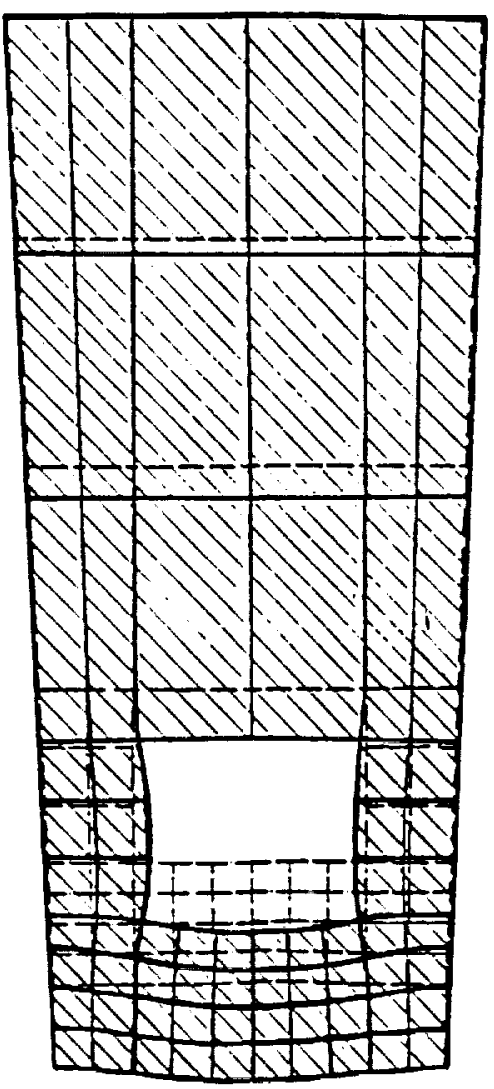

Figure 13: Deformed Geometry of the Cylindrical Thrust Chamber 


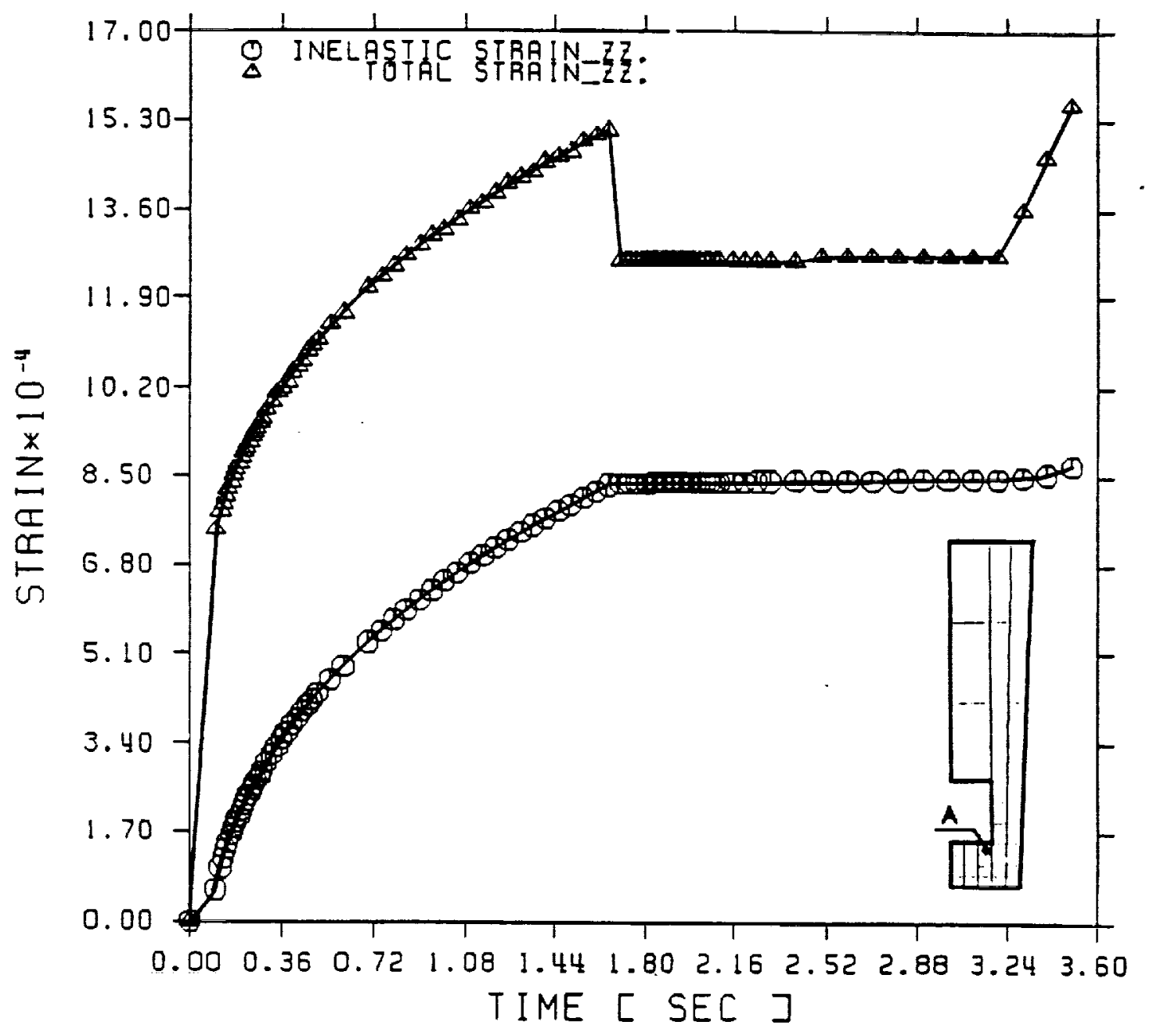

Figure 14: Strain History $\left(\epsilon_{z z}\right)$ at Point $A$ in the Thrust Chamber Using Asymptotic Algorithm 
W. 
Public reporting burden for this collection of information ls estimated to average 1 hour per response, including the time for reviewing instructions, searching existing data sources. gathering and maintaining the data needed, and completing and reviewing the collection of intormation. Send commente nogerding this burden eattimate or any ofher aspect of this

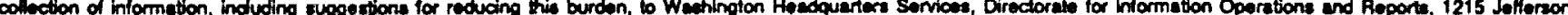
Davis Highway, Suite 1204, Alington, VA 22202-4302, and to the Office of Manapament and Budget, Papenwork Reduction Prolect (0704-0188), Washington, DC 20503.

\begin{tabular}{|l|l|l}
\hline 1. AOENCY USE ONLY (Leave blank) & $\begin{array}{c}\text { 2. REPOAT DATE } \\
\text { September } 1991\end{array}$ & $\begin{array}{r}\text { 3. AEPOAT TYPE AND DATES COVERED } \\
\text { Final Contractor Report }\end{array}$
\end{tabular}

4. TITLE AND SUETTTLE

Finite Element Implementation of State Variable-Based Viscoplasticity Models

5. FUNDINO NUMBERS
Finite Element Implementation of State Variable-Based Viscoplasticity Models

6. AUTHOA(S)

I. Iskovitz, T.Y.P. Chang, and A.F. Saleeb

WU - 510-01-50

G - NAG3 - 901

7. PERFORMING OAGANIZATION NAME(S) AND ADDRESS(ES)

6. PERForminio oroAnMzation REPORT NUMBER

University of Akron

Department of Civil Engineering

None

Akron, Ohio 44325

9. SPONSORING/MONTORING AGENCY MAMES(S) AND ADDRESS(ES)

National Aeronautics and Space Administration

Lewis Research Center

Cleveland, Ohio 44135 - 3191

10. SPONSORING/MONTORINO AGENCY REPORT NUMBER

NASA CR -187212

11. SUPPLEMENTARY NOTES

Project Manager, Steven M. Amold, Structures Division, NASA Lewis Research Center, (216) 433 - 3334.

12.. DISTRIBUTION/AVAILABILITY STATEMENT

12b. DISTRIBUTION CODE

Unclassified - Unlimited

Subject Category 39

13. ABSTAACT (Moximum 200 worda)

Implementation of state variable-based viscoplasticity models is made in a general purpose finite element code for structural applications of metals deformed at elevated temperature. Two constitutive models, i.e., Walker's and Robinson's models, are studied in conjunction with two implicit integration methods: the trapezoidal rule with NewtonRaphson iterations and an asymptotic integration algorithm. A comparison is made between the two integration methods, and the latter method appears to be computationally more appealing in terms of numerical accuracy and CPU time. However, in order to make the asymptotic algorithm robust, it is necessary to include a self adaptive scheme with subincremental step control and error checking of the Jacobian matrix at the integration points. Three examples are given to illustrate the numerical aspects of the integration methods tested.

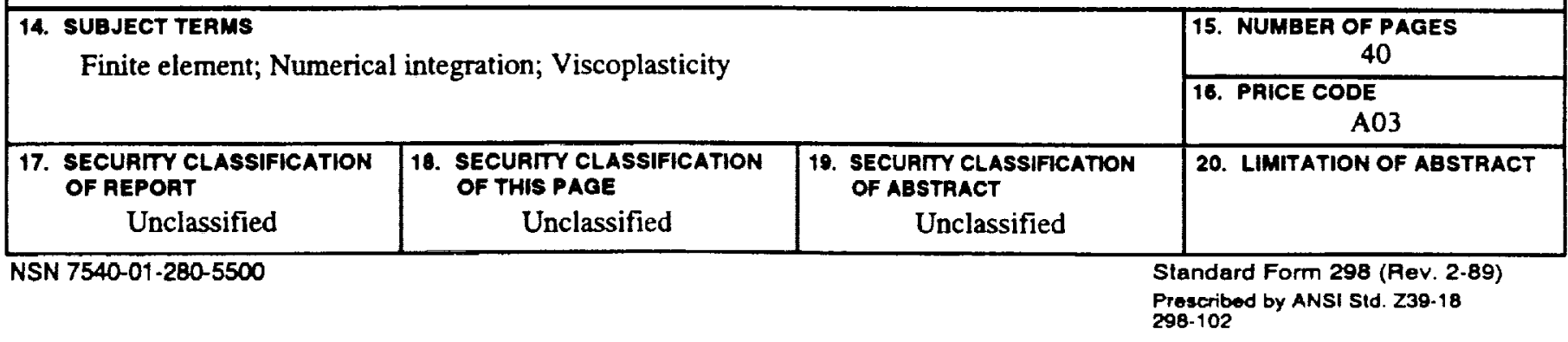

